\title{
Control of Allergic Rhinitis and Asthma Test (CARAT): dissemination and applications in primary care
}

\author{
Pedro Azevedo', Jaime Correia-de-Sousa², Jean Bousquet ${ }^{3}$, António Bugalho-Almeida ${ }^{4}$, \\ Stefano R Del Giacco ${ }^{5}$, Pascal Demoly ${ }^{6}$, Tari Haahtela ${ }^{7}$, Tiago Jacinto ${ }^{8}$, \\ Vanessa Garcia-Larsen ${ }^{9}$, Thys van der Molen ${ }^{10}$, Mário Morais-Almeida"1, \\ Luis Nogueira-Silva ${ }^{12}$, Ana M Pereira ${ }^{13}$, Miguel Román-Rodrígues ${ }^{14}$, Bárbara G Silva ${ }^{15}$, \\ loanna G Tsiligianni ${ }^{16}$, Hakan Yaman ${ }^{17}$, Barbara Yawn ${ }^{18}$, *João A Fonseca ${ }^{19}$ (in collaboration \\ with the WHO Collaborative Center for Asthma and Rhinitis, Montpellier)
}

\footnotetext{
1 Fânzeres Family Health Unit, Gondomar, Portugal

2 Life and Health Sciences Research Institute (ICVS), School of Health Sciences, University of Minho, Braga, Portugal, ICVS-3Bs - PT Government Associate Laboratory, Braga, Guimarães, Portugal and Horizonte Family Health Unit, Matosinhos, Porto, Portugal

${ }^{3}$ University Hospital Montpellier, Chairman of the Global Alliance against Chronic Respiratory Diseases (GARD)

${ }^{4}$ Centro de Imunoalergologia CUF, Lisboa, Portugal

${ }^{5}$ Department of Medical Sciences "M. Aresu", University of Cagliari, Italy

${ }^{6}$ University Hospital, Hôpital Arnaud de Villeneuve, Department of Respiratory Diseases, Montpellier, France

7 Department of Allergy, Skin and Allergy Hospital, Helsinki University Hospital, Helsinki, Finland

${ }^{8}$ CINTESIS and Health Information and Decision Sciences Department, Faculdade de Medicina da Universidade do Porto, Centro de Imunoalergologia CUF, Porto, Portugal

${ }^{9}$ National Heart and Lung Institute, Imperial College London, Royal Brompton and Harefield Foundation NHS Trust, London, UK

${ }^{10}$ Department of General Practice, University Medical Centre of Groningen, UMCG, The Netherlands ${ }^{2}$ Life and Health Sciences Research Institute (ICVS), SC

"Centro de Imunoalergologia, Hospital CUF Descobertas, Lisboa, Portugal

${ }^{12}$ Health Information and Decision Sciences Department, Faculdade de Medicina da Universidade do Porto, Porto, Portugal

${ }^{13}$ Health Information and Decision Sciences Department, Faculdade de Medicina da Universidade do Porto; Serviço de Imunoalergologia, Centro Hospitalar S. João, Porto, Portugal

${ }^{14}$ Son Pisa Primary Care Health Centre, IB Salut, Palma de Mallorca, Spain

${ }^{15}$ Hospital do Servidor Público Estadual de São Paulo, Allergy and Immunology Section, São Paulo, Brazil

${ }^{16}$ Agia Barbara Health Care, Heraklion, Crete, Greece and Department of General Practice, University Medical Centre of Groningen, UMCG, The Netherlands

${ }^{17}$ Akdeniz University, Department of Family Medicine, Antalya, Turkey

${ }^{18}$ Department of Research, Olmsted Medical Center, Rochester, Minnesota, USA

${ }^{19}$ CINTESIS and Health Information and Decision Sciences Department, Faculdade de Medicina da Universidade do Porto; Serviço de Imunoalergologia, Centro Hospitalar S. João; Centro de Imunoalergologia CUF, Porto, Portugal
}

Received 29th October 2012; revised 23rd November 2012; accepted 27th November 2012; online 14th February 2013

\begin{abstract}
Asthma frequently occurs in association with allergic rhinitis and a combined management approach has been suggested. The Control of Allergic Rhinitis and Asthma Test (CARAT) is the first questionnaire to assess control of both diseases concurrently. However, to have an impact on healthcare it needs to be disseminated and adopted. In this paper we discuss the dissemination of CARAT in different countries and its possible applications in primary care. At present, the adaptation of CARAT for use in different languages and cultures is being led by volunteer researchers and clinicians in 15 countries. Website and smartphone applications have been developed, and a free open model of distribution was adopted to contribute to the dissemination of CARAT. Examples of dissemination activities include distribution of leaflets and posters, educational sessions on the use of the questionnaire in the follow-up of patients, development of
\end{abstract}

\footnotetext{
* Corresponding author: Dr João A Fonseca, Faculdade de Medicina da Universidade do Porto, Ciências de Informacao e decisao em saúde, Al. Hernani Monteiro, 1, 4200-186 Porto, Portugal. Tel: +351914767661 E-mail: fonseca.ja@gmail.com
} 
clinical studies, collaborations with professional organisations and health authorities, and the inclusion of CARAT in clinical guidelines. The adoption of innovations is an important challenge in healthcare today, and research on the degree of success of dissemination strategies using suitable methods and metrics is much needed. We propose that CARAT can be used in a range of settings and circumstances in primary care for clinical, research and audit purposes, within the overall aim of increasing awareness of the level of disease control and strengthening the partnership between patients and doctors in the management of asthma and rhinitis.

(C) 2013 Primary Care Respiratory Society UK. All rights reserved.

P Azevedo et al. Prim Care Respir J 2013; 22(1): 112-116

http://dx.doi.org/10.4104/pcrj.2013.00012

Keywords asthma, rhinitis, control, questionnaire, dissemination, cultural adaptation

The full version of this paper, with online appendix,

is available online at www.thepcrj.org

\section{Introduction}

Asthma is a heterogeneous syndrome with multiple phenotypes, the most prevalent of which is allergic asthma in association with allergic rhinitis. ${ }^{1}$ The World Health Organization (WHO) recognises the close relationship between asthma and rhinitis through the Allergic Rhinitis and its Impact on Asthma (ARIA) ${ }^{2}$ guidelines and the Global Alliance against Chronic Respiratory Diseases (GARD) inititative. ${ }^{3}$ This recognition has resulted in a call for a change in asthma management and led to a combined approach for asthma and its comorbidities, particularly allergic rhinitis. ${ }^{4-7}$ Such an approach, however, needs simple assessment tools that can be readily used in clinical practice, and in primary care in particular. ${ }^{5,8}$

In 2007 a group of Portuguese allergologists, respiratory physicians, family physicians, and paediatricians identified the need for a questionnaire to assess asthma and allergic rhinitis control simultaneously. The initial goal of the Control of Allergic Rhinitis and Asthma Test (CARAT) project was to develop a questionnaire that would meet the needs of both patients and physicians. The questionnaire, which was designed to be self-administered by adults with a prior diagnosis of asthma and rhinitis, was developed and evaluated in three stages using different methodologies. We have described these stages elsewhere. ${ }^{9-11}$

The CARAT questionnaire is composed of 10 questions that address upper and lower airway symptoms, sleep interference, activity limitation, and the need to increase medication over a fourweek period (see Appendix 1, available online at www.thepcrj.org). The answers are rated on a four-point scale, with a total possible score ranging from 0 (minimum control) to 30 (maximum control). The properties of the questionnaire are summarised in Table 1. The questionnaire meets nine of the 10 items on the COSMIN (COnsensus-based Standards for the selection of health Measurement INstruments) checklist for evaluating the methodological quality of studies on measurement properties of patient-reported outcomes. ${ }^{12}$

The use of a formal methodological approach in the development of the questionnaire and the good measurement properties observed in the validation studies suggest that CARAT can be used in both clinical studies and clinical practice, allowing comparison between groups and the evaluation of individual patients over time.
Table 1. CARAT characteristics and properties

\begin{tabular}{|c|c|c|}
\hline & Instrument & CARAT \\
\hline \multirow{7}{*}{$\begin{array}{l}\stackrel{+}{0} \\
\stackrel{ \pm}{ \pm} \\
\stackrel{0}{0}\end{array}$} & Symptom frequency & Yes \\
\hline & Rescue therapy use & Yes \\
\hline & Sleep interference & Yes \\
\hline & Activity limitation & Yes \\
\hline & Exacerbations & No \\
\hline & Physiologic measures & No \\
\hline & Other & No \\
\hline \multirow{9}{*}{ 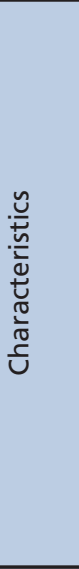 } & $\begin{array}{l}\text { Number of questions } \\
\text { Response format }\end{array}$ & $\begin{array}{l}10 \\
\text { 4-point Likert scale }\end{array}$ \\
\hline & Scoring method & Score sum 0-30 \\
\hline & Target population; age & $\geq 18$ years \\
\hline & Time to complete & $<3 \min$ \\
\hline & Patient report? & Yes \\
\hline & How is it administered? & $\begin{array}{l}\text { Self-administered (paper, Internet, } \\
\text { smart phone) }\end{array}$ \\
\hline & Recall period & 4 weeks \\
\hline & Languages & $\begin{array}{l}\text { Portuguese (PT), French, Turkish, } \\
\text { Italian, Dutch, Portuguese (BR), } \\
\text { English (UK), Spanish, Greek, } \\
\text { English (US), German, Swedish, } \\
\text { Finnish, Slovenian, Indian }\end{array}$ \\
\hline & Cost to use & FREE for clinical use \\
\hline \multirow{8}{*}{ 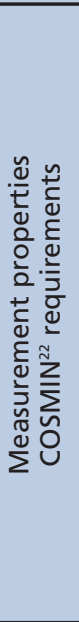 } & $\begin{array}{l}\text { Internal consistency } \\
\text { Reliability }\end{array}$ & $\begin{array}{l}\text { Cronbach's alpha was } 0.85^{10} \\
\text { ICC } 0.82^{11}\end{array}$ \\
\hline & Content validity & Face and content 9,10 \\
\hline & $\begin{array}{l}\text { Criterion validity } \\
\text { Hypothesis-testing }\end{array}$ & $\begin{array}{l}\text { Met a priori prediction for } \\
\text { correlation coefficients ranging } \\
\text { from } 0.58 \text { to } 0.79 ; \text {; AUC } 0.82{ }^{10}\end{array}$ \\
\hline & Structural validity & $\begin{array}{l}\text { Assessed using exploratory factor } \\
\text { analysis }{ }^{10}\end{array}$ \\
\hline & Cross-cultural validity & See text \\
\hline & Floor and ceiling effects & Not present ${ }^{10}$ \\
\hline & Responsiveness & $\begin{array}{l}\text { Significant within-patient change of } \\
\text { CARAT }^{10} \text { scoring in clinically } \\
\text { unstable patients ( } 95 \% \text { confidence } \\
\text { interval [-5.08; }-1.31], p=0.002 \text { ). } \\
\text { The Guyatt's responsiveness index } \\
\text { was } 1.54^{11}\end{array}$ \\
\hline & Interpretability & Clinically meaningful ${ }^{11}$ \\
\hline \multirow{2}{*}{ 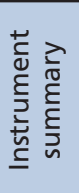 } & Main advantage & $\begin{array}{l}\text { Simple, simultaneous evaluation of } \\
\text { asthma and rhinitis in accordance } \\
\text { with ARIA guidelines }\end{array}$ \\
\hline & $\begin{array}{l}\text { Additional information } \\
\text { needed }\end{array}$ & $\begin{array}{l}\text { Minimal important difference } \\
\text { Sub score cut-off values clinical } \\
\text { validation of electronic versions }\end{array}$ \\
\hline
\end{tabular}


While questionnaires have been developed to assess the level of control of asthma ${ }^{13,14}$ and more recently of rhinitis, ${ }^{15,16}$ CARAT is the only questionnaire that concurrently assesses the control of both disorders. This concurrent approach has previously been used to assess disease-specific quality of life in the RHINASTHMA questionnaire. ${ }^{17}$ CARAT has recently been recognised by the ARIA group as the first tool to help implement ARIA guidelines in primary care. ${ }^{3}$ CARAT also meets the demand for a simple, feasible, and acceptable tool for assessing asthma and/or rhinitis in primary care. The need for such a tool was underlined in the recent International Primary Care Respiratory Group (IPCRG) respiratory research needs statement ${ }^{18}$ and in the group's analysis of the prioritisation of these needs. ${ }^{19}$

In this paper we discuss the dissemination of CARAT in different countries and its possible applications in primary care.

\section{Importance of dissemination}

One of the challenges facing medicine today is the quick and efficient translation of evidence into practice. ${ }^{20}$ Dissemination refers to a set of planned efforts designed to make a tool, technology, programme, or innovation more widely available..21 The use of different approaches can help to increase awareness and use among target populations. Moreover, new tools, technologies, and resources need to be easily accessible and simple to use in different settings and situations. If CARAT is to be successfully implemented in primary care, its widespread availability is therefore crucial.

\section{Cross-cultural adaptations}

The ARIA group has recognised the need rapidly to test CARAT in different languages to facilitate the implementation of this tool. ${ }^{3}$ Moreover, numerous researchers, many of whom are members of the IPCRG, have expressed their interest in CARAT. The use of this questionnaire in international settings, however, requires prior translation and cross-cultural adaptation.

To assist in this task, the CARAT team has developed a protocol for the translation and cross-cultural adaptation of the questionnaire based on international recommendations and best practices. ${ }^{22-24}$ Essentially, researchers interested in developing versions for their country/language are invited to participate in the process, which is described in detail at http://caratnetwork.org, a dedicated website which also contains a web tool to help researchers follow the stipulated protocol.

The protocol has three main steps: forward translation, back translation, and patient testing. In the forward translation step, two people independently translate the original Portuguese questionnaire into the target language. The translations are then compared and an agreed version is drawn up between the translators and the CARAT team members (first version). In the back translation step, the text is translated back into Portuguese with the support of the CARAT team. It is then compared with the original and reviewed to ensure conceptual equivalence. Written feedback is given to the researchers who produce a revised second version. The last step is the patient test phase. Ten adult patients are given the second version of CARAT and interviewed about the interpretation and wording of each item. During this phase the researchers make
Table 2. Current status of the CARAT cross-cultural and language adaptation process

\begin{tabular}{lll} 
Country & Phase & Investigators \\
\hline France & Completed & Demoly and Pereira \\
\hline Turkey & Completed & Yaman \\
\hline Italy & Completed & Del Giacco \\
\hline The Netherlands & Completed & van der Molen and Tsiligianni \\
\hline Greece & Completed & Tsiligianni \\
\hline UK & PT & Garcia-Larsen \\
\hline Brazil & PT & Gonçalves da Silva \\
\hline Spain & PT & Román-Rodrígues and Mascarós \\
\hline USA & BT & Yawn \\
\hline Finland & BT & Haahtela and Pereira \\
\hline Switzerland & FT & Under appraisal \\
\hline Germany & FT & \\
\hline Slovenia & FT & \\
\hline India & FT & \\
\hline
\end{tabular}

$\mathrm{FT}=$ forward translation, $\mathrm{BT}=$ back translation, $\mathrm{PT}=$ patient testing .

a note of the number of individuals interviewed, their age, the time taken to complete the questionnaire, and any difficulties encountered and solutions proposed. The results are reviewed and any changes are integrated into the third and final version of the questionnaire.

To date, research groups from 15 countries have participated in cross-cultural adaptations of CARAT. Many of the researchers involved are members of the IPCRG. The current status of countryspecific versions of the questionnaire is shown in Table 2. Typically, researchers need around 12 hrs of work to complete the crosscultural adaptation process and between 4 and 12 weeks are required to produce the CARAT for a new language/ country/population. This time includes approval from a review board, a back translation report, and feedback from the CARAT team after the patient test phase.

CARAT can be used after completion of the cross-cultural adaptation, but clinical validation studies would improve the knowledge on how CARAT performs. The CARAT team has also designed a study protocol that can be used by researchers wishing to carry out clinical validation studies in their countries.

\section{CARAT for children}

A CARAT questionnaire for children aged 6-12 years is currently under development. The first version was completed in December 2011 after consensus meetings and cognitive interviews with children and their parents, and the questionnaire is currently being evaluated in a prospective validation study.

\section{CARAT-NETWORK and $m$ CARAT}

A website and smartphone applications have been developed to contribute to the dissemination of CARAT. In addition to providing support for cross-cultural adaptations, the CARAT-NETWORK (http://caratnetwork.org) provides access to the different formats and versions of the questionnaire. Users can complete the 
questionnaire online and print the results. Paper versions can also be downloaded, and links to the smartphone applications (Android and iOS operative systems) are available. After free online registration, users gain access to additional tools, which include resources for plotting and sharing the results of CARAT.

The main features of the smartphone application (mCARAT) have already been reported elsewhere. ${ }^{25}$ Briefly, the patient can fill in the questionnaire, view and email the results, and synchronise with the CARAT-NETWORK website. The Android OS version of the application allows the user to set alarms for tasks such as taking medications and keeping medical appointments, and also includes features for recording symptoms, exacerbations, medication use, and scheduled or unscheduled medical visits, as well as lung function test results. Additionally, mCARAT allows customised access to online educational resources about asthma and rhinitis. The mCARAT application will shortly undergo usability and clinical usefulness evaluation.

\section{Dissemination experiences}

In Portugal, leaflets and posters with the questionnaire have been distributed in primary healthcare centres, and 80 educational sessions on the use of the questionnaire in the follow-up of patients with asthma and rhinitis have been held with the participation of over 1200 physicians, nurses, and pharmacists. Furthermore, the Portuguese Government recommended CARAT for the follow-up of patients in the 2011 asthma guidelines.

In the Netherlands the cross-cultural adaptation has been completed and further research is underway to assess asthma and rhinitis control in the Dutch population during the allergy season.

In the UK, the standard operational procedure for translation of CARAT into English has been completed and the project is currently being reviewed for ethical approval at Imperial College London.

A Spanish version has also been developed for use in Spain and Spanish-speaking countries. The questionnaire is now awaiting clinical validation.

In Italy, the cross-cultural and language adaptation process has been successfully completed, and preparations are being made in collaboration with the Italian General Practitioners' Medical Society for a preliminary evaluation of the use of CARAT in primary care.

The Greek version of the questionnaire has also been produced, with results showing that it is easy to complete for both clinicians and patients. Further dissemination is anticipated within the Greek Association of General Practitioners (ELEGEIA) and the Clinic of Social and Family Medicine of the University of Crete.

The Brazilian version of CARAT is under clinical validation. Unpublished preliminary data indicate that it may increase compliance with treatment, and understanding of disease, among Brazilian patients

\section{Use case scenarios in primary care}

Currently, CARAT can be used to assess disease control in adults with a prior diagnosis of asthma and rhinitis in a range of settings and circumstances (Figure 1). These include follow-up consultations (general practice or allergy/asthma outpatient clinics), nurse

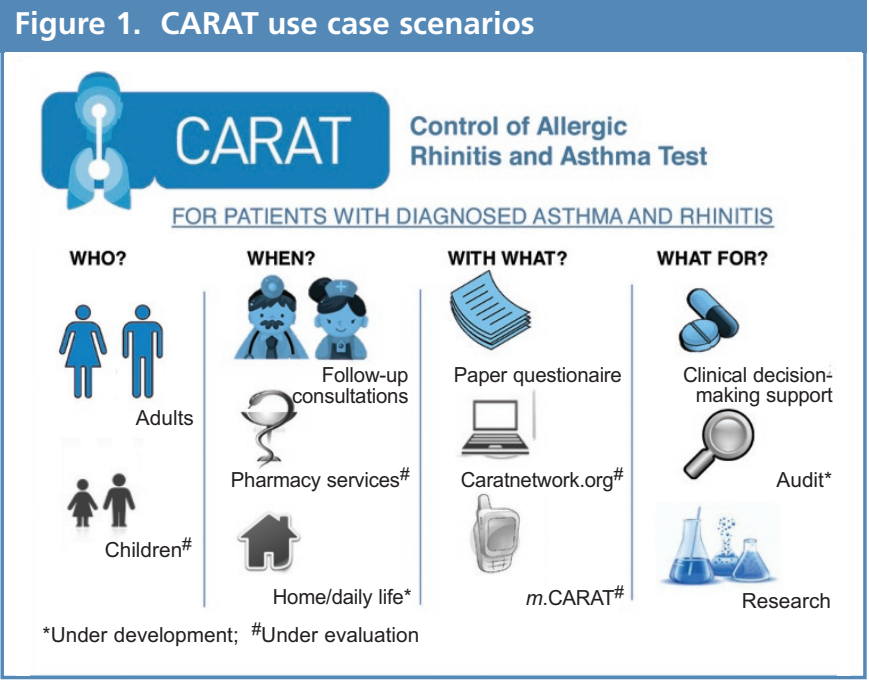

consultations, pharmacy services, lung function laboratories, and patient self-monitoring.

The information generated by the questionnaire has three broad applications: (1) by providing an objective measure of disease control, it can be used to support clinical decision-making and increase patient awareness of their level of asthma/rhinitis control; (2) it can be used for patient-reported outcome assessments in asthma/rhinitis research; and (3) it can be used to assess and improve the quality of asthma/rhinitis care and may have a role in the benchmarking of healthcare quality.

By increasing patient awareness of their level of asthma/rhinitis control and enhancing patient empowerment, CARAT has the potential to contribute to strengthening the much needed partnership between patients and doctors. In this regard, we believe that the involvement of patient organisations in the dissemination of the questionnaire is to be encouraged. The fact that the questionnaire is freely and easily available in various formats should encourage its use among patients and help to engage them in their own care.

\section{Questions for the future}

The dissemination and adoption of any healthcare innovation is a long and difficult process, with uncertain outcomes. Evaluation of dissemination strategies should thus become a standard component of this process, but equally important are suitable methods and metrics to evaluate the effectiveness of innovations in clinical practice.

Future research in primary care settings needs to investigate whether the combined assessment of rhinitis and asthma control during routine clinical visits reduces the risk of exacerbations, emergency room attendances, and hospital admissions and improves patients' quality of life. It also needs to assess how - and how often - CARAT results change clinical decisions made by physicians in the routine assessment of asthma and how it changes the patient's understanding and adherence to the physician's therapeutic decision.

Moreover, to ensure that CARAT meets all the quality criteria of COSMIN, analysis of large longitudinal datasets is needed to establish the minimal clinically important difference of the 
questionnaire and the definitive cut-off values for the CARAT overall score and subscores. It is within the scope of this research group to provide these answers in the near future.

\section{Conclusions}

CARAT is the first questionnaire to assess control of asthma and allergic rhinitis concurrently. However, to have an impact on healthcare it needs to be disseminated and adopted. The dissemination approaches used were: (1) widespread availability (cross-cultural adaptations, print, web, and mobile versions); (2) a free open model of distribution; (3) user support through a dedicated website; (4) clinical educational sessions; and (5) dialogue with health authorities and integration into clinical guidelines.

At present the adaptation of CARAT for use in different languages and cultures is being led by volunteer researchers and clinicians in 15 countries. Studying the degree of success of dissemination strategies using suitable methods and metrics is much needed as adoption of innovations is an important challenge in healthcare today.

We propose that CARAT be used in primary care for clinical, research, and audit purposes, within the overall aim of strengthening the partnership between patients and doctors in the management of asthma and rhinitis.

\section{Handling editor Mike Thomas}

Acknowledgements We would like to thank all those involved in the development and dissemination of CARAT, particularly Eduardo Burnay and André Ribeiro for their work on mCARAT app and caratnetwork.org, Ana Sá e Sousa for help with the CARAT project, and Ricardo Monteiro for believing in and supporting the CARAT project.

Conflicts of interest The authors declare that they have no conflicts of interest in relation to this article.

Contributorship PA wrote the first draft of the paper. JCS conceived the idea for the manuscript and wrote parts of the manuscript. TJ, AMP and LNS wrote parts of the manuscript. SRG, VGL, TVdM, MRR and BGS participated in the cross-cultural adaptations, participated in writing the dissemination experiences section and reviewed the manuscript. PD, TH, IGT, HY and BY participated in the cross-cultural adaptations and reviewed the manuscript. JB and ABA provided critical review and reviewed the manuscript. JAF and MMA are responsible for the CARAT project and participated in all stages and tasks. All authors have read and approved the final manuscript.

Funding No specific funding was received for writing this paper. The CARAT project has received unrestricted grants from Sociedade Portuguesa de Alergologia e Imunologia Clínica, Merck Sharp Dohme Portugal and Associação Portuguesa de Asmáticos e Alérgicos.

\section{References}

1. Wenzel S. Asthma: defining of the persistent adult phenotypes. Lancet 2006;368(9537):804-13. http://dx.doi.org/10.1016/50140-6736(06)69290-8

2. Cruz AA, Popov T, Pawankar R, et al. ARIA Initiative Scientific Committee. Common characteristics of upper and lower airways in rhinitis and asthma: ARIA update, in collaboration with GA(2)LEN. Allergy 2007;62(Suppl 84):1-41. http://dx.doi.org/ 10.1111/j.1398-9995.2007.01551.x

3. Bousquet J, Schünemann HJ, Zuberbier T, et al. Development and implementation of guidelines in allergic rhinitis - an ARIA-GA2LEN paper. Allergy 2010;65(10):1212-21. http://dx.doi.org/10.1111/j.1398-9995.2010.02439.x

4. Price D, Zhang Q, Kocevar VS, Yin DD, Thomas M. Effect of a concomitant diagnosis of allergic rhinitis on asthma-related healthcare use by adults. Clin Exp Allergy 2005;35(3):282-7. http://dx.doi.org/10.1111/j.1365-2222.2005.02182.x

5. Costa DJ, Bousquet PJ, Ryan D, et al. Guidelines for allergic rhinitis need to be used in primary care. Prim Care Respir J 2009;18(4):250-7. http://dx.doi.org/10.4104/ pcrj.2009.00028

6. Andrade CR, Cunha Ibiapina C, Gonçalves AC, Fernandes FMJ, Lima BLM, Moreira PA. Asthma and allergic rhinitis co-morbidity: a cross-sectional questionnaire study on adolescents aged 13-14 years. Prim Care Respir J 2008;17(4):222-5. http://dx.doi.org/10.3132/pcri.2008.00056

7. Holgate S, Bjermer L, Haahtela T, et al. The Brussels Declaration: the need for change in asthma management. Eur Respir J 2008;32(6):1433-42. http://dx.doi.org/ 10.1183/09031936.00053108

8. Pinnock $\mathrm{H}$, Fletcher $\mathrm{M}$, Holmes $\mathrm{S}$, et al. Setting the standard for routine asthma consultations: a discussion of the aims, process and outcomes of reviewing people with asthma in primary care. Prim Care Respir J 2010;19(1):75-83. http://dx.doi.org/10.4104/pcri.2010.00006

9. Nogueira-Silva L, Martins SV, Cruz-Correia R, et al. Control of allergic rhinitis and asthma test: a formal approach to the development of a measuring tool. Respir Res 2009;10:52. http://dx.doi.org/10.1186/1465-9921-10-52

10. Fonseca JA, Nogueira-Silva L, Morais-Almeida $\mathrm{M}$, et al. Validation of a questionnaire (CARAT10) to assess rhinitis and asthma in patients with asthma. Allergy 2010;65(8):1042-8. http://dx.doi.org/10.1111/.1398-9995.2009.02310.x

11. Fonseca JA, Nogueira-Silva L, Morais-Almeida M, et al. Control of Allergic Rhinitis and Asthma Test (CARAT) can be used to assess individual patients over time. Clin Transl Allergy 2012;2(1):16. http://dx.doi.org/10.1186/2045-7022-2-16.

12. Mokkink $L B$, Terwee $C B$, Knol DL, et al. The COSMIN checklist for evaluating the methodological quality of studies on measurement properties: a clarification of its content. BMC Med Res Methodol 2010;10:22. http://dx.doi.org/10.1186/1471 2288-10-22

13. Nathan RA, Sorkness CA, Kosinski M, et al. Development of the asthma control test: a survey for assessing asthma control. J Allergy Clin Immunol 2004;113(1):59-65. http://dx.doi.org/10.1016/j.jaci.2003.09.008

14. Juniper EF, O'Byrne PM, Guyatt GH, Ferrie PJ, King DR. Development and validation of a questionnaire to measure asthma control. Eur Respir J 1999;14:902-07. http://dx.doi.org/10.1034/j.1399-3003.1999.14d29.x

15. Demoly $P$, Jankowski $R$, Chassany $O$, Bessah $Y$, Allaert FA. Validation of a selfquestionnaire for assessing the control of allergic rhinitis. Clin Exp Allergy 2011;41(6):860-8. http://dx.doi.org/10.1111/j.1365-2222.2011.03734.x

16. Schatz M, Meltzer EO, Nathan R, et al. Psychometric validation of the Rhinitis Control Assessment Test: a brief patient-completed instrument for evaluating rhinitis symptom control. Ann Allergy Asthma Immunol 2010;104(2):118-24. http://dx.doi.org/10.1016/..anai.2009.11.063

17. Baiardini I, Pasquali M, Giardini A, et al. Rhinasthma: a new specific QoL questionnaire for patients with rhinitis and asthma. Allergy 2003;58(4):289-94. http://dx.doi.org/10.1034/j.1398-9995.2003.00079.x

18. Pinnock $H$, Thomas $M$, Tsiligianni I, et al. The International Primary Care Respiratory Group (IPCRG) Research Needs Statement 2010. Prim Care Respir J 2010;19(Suppl 1):S1-20. http://dx.doi.org/10.4104/pcri.2010.00021

19. Pinnock $H, \varnothing s t r e m ~ A$, Rodriguez MR, et al. Prioritising the respiratory research needs of primary care: the International Primary Care Respiratory Group (IPCRG) e-Delphi exercise. Prim Care Respir J 2012;21(1):19-27. http://dx.doi.org/10.4104/ pcrj.2012.00006

20. Lenfant C. Clinical research to clinical practice - lost in translation? N Engl I Med 2003;349:868-74. http://dx.doi.org/10.1056/NEJMsa035507

21. Owen N, Glanz K, Sallis JF, Kelder SH. Evidence-based approaches to dissemination and diffusion of physical activity interventions. Am J Prev Med 2006;31(4 Suppl):S3544. http://dx.doi.org/10.1016/j.amepre.2006.06.008

22. Wild D, Grove A, Martin M, et al; ISPOR Task Force for Translation and Cultural Adaptation. Principles of good practice for the translation and cultural adaptation process for patient-reported outcomes (PRO) measures: report of the ISPOR Task Force for Translation and Cultural Adaptation. Value Health 2005;8(2):94-104. http://dx.doi.org/10.1111/j.1524-4733.2005.04054.x

23. Braido F, Bousquet PJ, Brzoza Z, et al. Specific recommendations for PROs and HRQoL assessment in allergic rhinitis and/or asthma: a GA(2)LEN taskforce position paper. Allergy 2010;65:959-68; Annex III 953.

24. Tomlins R. International Primary Care Respiratory Group (IPCRG) guidelines: dissemination and implementation - a proposed course of action. Prim Care Respir J 2006;15(1):71-4. http://dx.doi.org/10.1016/j.pcrj.2005.11.004

25. Burnay E, Jacinto T, Sa-Sousa A, Cruz-Correia R, Fonseca J. Challenges of a mobile application for asthma and allergic rhinitis patient enablement - interface and synchronization. Telemed e-Health 2013 (in press). 


\section{Appendix 1. Control of Allergic Rhinitis and Asthma Test}

Please mark the following boxes with a cross ( $\mathbf{~ ) . ~}$

Due to your allergic respiratory diseases (asthma, rhinitis, allergies) in the last four weeks, on average, how many times did you have:

$\begin{array}{ccc}\text { Never } & \begin{array}{c}1 \text { or } 2 \text { days } \\ \text { per week }\end{array} & \text { More than } 2 \\ & \text { days per week }\end{array}$

\section{Almost every day}

\section{Blocked nose?}

$\square$

$\square^{2}$

$\square 1$

$\square$ 。

\section{Sneezing?}

$\square^{3}$

$\square^{2}$

$\square^{1}$

$\square \circ$

\section{Itchy nose?}

4. Runny nose?

$\square^{3}$

$\square^{2}$

$\square^{1}$

$\square$ 。

4. Runny nose? $\square^{3} \quad \square^{2} \quad \square^{1} \quad \square$ 。

5. Shortness of breath/dyspnoea?

$\square^{3} \quad \square^{2} \quad \square^{1} \quad \square$ 。

6. Wheezing in the chest?

$\square^{3}$

$\square^{1}$

$\square \circ$

\section{Chest tightness upon physical} exercise?

8. Tiredness/ limitations in doing daily

tasks because of your allergic

$\square^{3} \quad \square^{2}$

$\square^{1}$

$\square$

respiratory diseases?

9. Woke up during the night because of your allergic respiratory diseases?

In the last 4 weeks, because of your allergic respiratory diseases (asthma, rhinitis, allergies), how many times did you:

1. increased the use (dosage or frequency) of your medicines?

$\begin{array}{cccc}\text { I'm not taking any } & \text { Never } & \text { Less than } & 7 \text { or more } \\ \text { medicines } & 7 \text { days } & \text { days }\end{array}$

$\square 0$ . 